九州大学学術情報リポジトリ

Kyushu University Institutional Repository

\title{
MOMENTS OF SOME STATISTICS OF PITMAN SAMPLING FORMULA
}

\author{
Yamato, Hajime \\ Department of Mathematics and Computer Science, Kagoshima University \\ Shibuya, Masaaki \\ Takachiho University
}

https://doi.org/10.5109/13490

出版情報: Bulletin of informatics and cybernetics. 32 (1), pp.1-10, 2000-06. Research Association of Statistical Sciences

バージョン :

権利関係 : 


\title{
MOMENTS OF SOME STATISTICS OF PITMAN SAMPLING FORMULA
}

\author{
By
}

\author{
Hajime Yamato* and Masaaki SibuYa ${ }^{\dagger}$
}

\begin{abstract}
For Pitman sampling formula which is a distribution on all unordered partitions of a positive integer, we give the moments of the number of components of the random partition which are the descending factorial moments, the ascending factorial moments and the usual moments. Using them the asymptotic distribution of the number of components of the random partition is derived. The moments of Pitman sampling formula are also given. Using them we give the asymptotic distributions of the marginals and their functions.
\end{abstract}

Key Words and Phrases: Random partition, Pitman sampling formula, MittagLeffler distribution.

\section{Introduction}

Let $\mathcal{M}_{n}$ denote the set of all unordered partitions of a positive integer $n$, that is,

$$
\mathcal{M}_{n}=\left\{\left(m_{1}, \ldots, m_{n}\right): m_{i} \geq 0(i=1, \ldots, n) \text { and } \sum_{j=1}^{n} j m_{j}=n\right\} .
$$

As a probability distribution on $\mathcal{M}_{n}$, generaliving Ewens sampling formula Pitman (1995) derives the distribution given by

$$
P\left(\left(M_{1}, \ldots, M_{n}\right)=\left(m_{1}, \ldots, m_{n}\right)\right)=n ! \frac{\theta^{[k ; \alpha]}}{\theta^{[n]}} \prod_{j=1}^{n}\left(\frac{(1-\alpha)^{[j-1]}}{j !}\right)^{m_{j}} \frac{1}{m_{j} !},
$$

where $\left(m_{1}, \ldots, m_{n}\right) \in \mathcal{M}_{n}, x^{[j: \alpha]}=x(x+\alpha) \cdots(x+(j-1) \alpha), x^{[j]}=x^{[j: 1]}, k=\sum_{j=1}^{n} m_{j}$ the number of components of the partition, and $0 \leq \alpha<1, \theta>-\alpha$.

In this paper we consider Pitman sampling formula corresponding to sampling from an infinite population. Since the case of $\alpha=0$ gives Ewens sampling formula, we consider only the case of $0<\alpha<1$. The distribution of $K_{n}=\sum_{j=1}^{n} M_{j}$ is given by

$$
P\left(K_{n}=k\right)=\frac{\theta^{[k: \alpha]}}{\theta^{[n]}}|C(n, k, \alpha)| \alpha^{-k}, k=1,2, \ldots, n
$$

\footnotetext{
* Department of Mathematics and Computer Science, Kagoshima University, Kagoshima 890-0065, Japan

† Takachiho University, Suginamiku 168-8508, Japan
} 
where $|C(n, k, \alpha)|=(-1)^{n-k} C(n, k, \alpha)$ and $C(n, k, \alpha)$ is the generalized Stirling numbers (Pitman(1996b,1999), Yamato et al.(1999)).

In Section 2, we give the descending factorial moments of $K_{n}$. Since it is not easy to handle directly the moments based on the probability given by (2), we derive the descending factorial moments of $K_{n}$ by using their recurrence relation. Using the descending factorial moments of $K_{n}$ we give its usual moments and ascending factorial moments. In case of $\theta=0$, the ascending factorial moments are given by Pitman (1996b).

Propositions 4 and 6 of Pitman (1997) shows the almost sure convergence of $K_{n} / \pi^{\alpha}$ and Theorem 2.2 of Feng and Hoppe (1998) also gives it. Pitman (1996b) gives the almost sure convergence of $K_{n} / n^{\alpha}$, its convergence in the pth mean and its asymptotic distribution. By evaluating $C(n, k, \alpha)$ asymptotically, Pitman (1999) obtains the asymptotic distribution of $K_{n} / n^{\alpha}$. In Section 3 we shall show it using the method of moments. By taking the limits of the moments obtained in Section 2, we show the asymptotic distribution of $K_{n} / n^{\alpha}$.

In Section 4, the asymptotic mean, variance, covariance and distribution for marginals of Pitman sampling formula are given. For the number of components smaller than or equal to a fixed positive integer, we give its asymptotic distribution. By the property of the tails of the underlying distribution, these statistics also converge almost surely.

In Section 5 which is Appendix, we give the proofs of the usual moments and ascending factorial moments of $K_{n}$ stated in Section 2.

\section{Moments}

We consider the sequence of random variables $B_{1}(\equiv 1), B_{2}, B_{3}, \ldots$ such that for $j=1,2, \ldots$ and $b_{1}=1, b_{2}, \ldots, b_{j}=0,1$

$$
\begin{aligned}
& P\left(B_{j+1}=1 \mid B_{1}=b_{1}, \ldots, B_{j}=b_{j}\right)=\frac{\theta+\left(b_{1}+\cdots+b_{j}\right) \alpha}{j+\theta}, \\
& P\left(B_{j+1}=0 \mid B_{1}=b_{1}, \ldots, B_{j}=b_{j}\right)=\frac{j-\left(b_{1}+\cdots+b_{j}\right) \alpha}{j+\theta} .
\end{aligned}
$$

Then we can consider $K_{n}=B_{1}+\cdots+B_{n}$ for $n=1,2, \ldots$, and it holds that

$$
P\left(B_{n+1}=1 \mid K_{n}=k\right)=\frac{\theta+k \alpha}{\theta+n}, P\left(B_{n+1}=0 \mid K_{n}=k\right)=\frac{n-k \alpha}{\theta+n} .
$$

The sequence of random variables $\left\{B_{j}\right\}$ is, for example, constructed from the sample from the two-parameter GEM distribution (Yamato et al.(1999)). Generally, for the sequence of random variables $\left\{X_{n}\right\}$ subject to the two-parameter model with parameters $0 \leq \alpha<1$ and $\theta>-\alpha$ (Pitman (1995, 1996a)), we put $B_{1} \equiv 1$. For $j=1,2, \ldots$ we put $B_{j+1}=1$ if $X_{j+1} \neq X_{1}, \ldots, X_{j}$ and $B_{j+1}=0$ if $X_{j+1}$ is equal to any one of $X_{1}, \ldots, X_{j}$. This sequence of random variables $\left\{B_{j}\right\}$ satisfies the conditions (2.1) and (2.2).

Since $K_{n+1}=K_{n}+B_{n+1}$ with $B_{n+1}=0$ or 1 , we have $K_{n+1}^{(r)}=\left(K_{n}+B_{n+1}\right)^{(r)}=$ 
$K_{n}^{(r)}+r K_{n}^{(r-1)} B_{n+1}$ where $x^{(r)}=x(x-1) \cdots(x-r+1)$. Then by (2.3) we have $E\left[K_{n+1}^{(r)} \mid K_{n}\right]=K_{n}^{(r)}+r K_{n}^{(r-1)}\left[\theta+(r-1) \alpha+\left(K_{n}-r+1\right) \alpha\right] /(\theta+n)$. Taking the expectations of this conditional expectation, we have the following recursive relation about the descending factorial moment $E K_{n}^{(r)}$.

Lemma 2.1 For $r=1,2, \ldots$,

$$
E K_{n+1}^{(r)}=\left(1+\frac{\alpha r}{n+\theta}\right) E K_{n}^{(r)}+\frac{r[\theta+(r-1) \alpha]}{n+\theta} E K_{n}^{(r-1)}
$$

This relation can be also obtained from (14) of Pitman (1996b) with $f(k)=k^{(r)}$. For $r=1$, we have

$$
E K_{n+1}=\frac{\theta}{n+\theta}+\left(1+\frac{\alpha}{n+\theta}\right) E K_{n}
$$

((15) of Pitman (1996b)). Using this relation recursively, we have

$$
\begin{aligned}
E K_{n+1}= & \frac{\theta}{n+\theta}+\left(1+\frac{\alpha}{n+\theta}\right) \frac{\theta}{n-1+\theta}+\cdots \\
& +\left(1+\frac{\alpha}{n+\theta}\right)\left(1+\frac{\alpha}{n-1+\theta}\right) \cdots\left(1+\frac{\alpha}{1+\theta}\right) \cdot 1
\end{aligned}
$$

The right hand side of the above is equal to $(\theta / \alpha)\left[\left\{(\theta+\alpha)^{[n+1]} / \theta^{[n+1]}\right\}-1\right]$, which is easily verified by induction. Thus for $n=1,2, \ldots$ we have

$$
E K_{n}^{(1)}=E K_{n}=\frac{\theta}{\alpha}\left[\frac{(\theta+\alpha)^{[n]}}{\theta^{[n]}}-1\right]
$$

This is also given by (18) of Pitman (1996b) and can be read from a formula of Engen (1978) for the 2 parameter residual allocation model. In general, we have the following descending factorial moments given by (2.5) which is easily derived by induction on $n$ and $r$ using (2.4).

Proposition 2.2 For $n=1,2, \ldots$ and $r=1,2, \ldots$, the $r$ th descending factorial moment of $K_{n}$ is

$$
\begin{gathered}
E K_{n}^{(r)}=\frac{\theta^{[r: \alpha]}}{\alpha^{r} \theta^{[n]}} \sum_{j=0}^{r}(-1)^{r-j}\left(\begin{array}{c}
r \\
j
\end{array}\right)(\theta+j \alpha)^{[n]} \\
=\left(\frac{\theta}{\alpha}\right)^{[r]} \sum_{j=0}^{r}(-1)^{r-j}\left(\begin{array}{c}
r \\
j
\end{array}\right) \frac{(\theta+j \alpha)^{[n]}}{\theta^{[n]}}
\end{gathered}
$$

Especially in case of $r=2$,

$$
E K_{n}^{(2)}=\frac{\theta(\theta+\alpha)}{\alpha^{2} \theta^{[n]}}\left\{(\theta+2 \alpha)^{[n]}-2(\theta+\alpha)^{[n]}+\theta^{[n]}\right\} .
$$

Thus we have

$$
\operatorname{Var}\left(K_{n}\right)=\frac{\theta(\theta+\alpha)}{\alpha^{2}} \cdot \frac{(\theta+2 \alpha)^{[n]}}{\theta^{[n]}}-\frac{\theta^{2}}{\alpha^{2}}\left(\frac{(\theta+\alpha)^{[n]}}{\theta^{[n]}}\right)^{2}-\frac{\theta}{\alpha} \cdot \frac{(\theta+\alpha)^{[n]}}{\theta^{[n]}}
$$


For the ascending factorial moment $E K_{n}^{[r]}$, we note that $E K_{n+1}^{[r]}=E\left(K_{n}+r-1+\right.$ $\left.B_{n+1}\right)^{(r)}=E\left(K_{n}+r-1\right)^{(r)}+r E\left[\left(K_{n}+r-1\right)^{(r-1)}\left(\theta+K_{n} \alpha\right) /(\theta+n)\right]$. Thus we get the recursive relation

$$
E K_{n+1}^{[r]}=\left(1+\frac{r \alpha}{n+\theta}\right) E K_{n}^{[r]}+\frac{r \theta}{n+\theta} E\left(K_{n}+1\right)^{[r-1]},
$$

which can be also obtained from (14) of Pitman (1996b) with $f(k)=k^{[r]}$. In case of $\theta=0$, we have

$$
E K_{n+1}^{[r]}=\left(1+\frac{r \alpha}{n}\right) E K_{n}^{[r]} \text { and } E K_{n}^{[r]}=\frac{(r-1) !(r \alpha)^{[n]}}{(n-1) ! \alpha}
$$

which are given by Proposition 2 of Pitman (1996b).

For the usual moment $E K_{n}^{r}$, using the Stirling number of the second kind $\left\{\begin{array}{l}r \\ i\end{array}\right\}$ we can write $E K_{n}^{r}=\sum_{i=0}^{r}\left\{\begin{array}{c}r \\ i\end{array}\right\} E K_{n}^{(i)}$ and by the relation (2.5) we have the following.

Corollary 2.3 For $n=1,2, \ldots$ and $r=1,2, \ldots$

$$
E K_{n}^{r}=\sum_{j=0}^{r}(-1)^{r-j}\left(1+\frac{\theta}{\alpha}\right)^{[j]} R\left(r, j, \frac{\theta}{\alpha}\right) \frac{(\theta+j \alpha+1)^{[n-1]}}{(\theta+1)^{[n-1]}},
$$

where $R(r, j, \lambda)$ is the unique function satisfying

$$
\sum_{j=0}^{r} y^{(j)} R(r, j, \lambda)=(y+\lambda)^{r}
$$

for any $y, \lambda$ and $r=1,2, \ldots$.

This function $R(r, j, \lambda)$ is introduced by Carlitz (1980) and the relation (2.7) is (3.4) of Carlitz (1980). The moment (2.6) is shown in Appendix. For example,

$$
E K_{n}^{2}=\left(1+\frac{\theta}{\alpha}\right)^{[2]} \frac{(\theta+2 \alpha+1)^{[n-1]}}{(\theta+1)^{[n-1]}}-\left(1+\frac{\theta}{\alpha}\right)\left(1+2 \frac{\theta}{\alpha}\right) \frac{(\theta+\alpha+1)^{[n-1]}}{(\theta+1)^{[n-1]}}+\left(\frac{\theta}{\alpha}\right)^{2} \text {. }
$$

Using the unsigned Stirling number of the first kind $\left[\begin{array}{c}r \\ i\end{array}\right]$ we can write $E K_{n}^{[r]}=$ $\sum_{i=0}^{r}\left[\begin{array}{l}r \\ i\end{array}\right] E K_{n}^{i}$ and by the relation (2.6), in case of $\theta \neq 0$, we have the following.

Corollary 2.4 For $n=1,2, \ldots$ and $r=1,2, \ldots$,

$$
E K_{n}^{[r]}=\sum_{j=0}^{r}(-1)^{r-j}\left(\begin{array}{l}
r \\
j
\end{array}\right)\left(\frac{\theta}{\alpha}\right)^{[j]}\left(\frac{\theta}{\alpha}\right)^{(r-j)} \frac{(\theta+j \alpha)^{[n]}}{\theta[n]}, \theta \neq 0 .
$$

This is also shown in Appendix. For example,

$$
E K_{n}^{[2]}=\left(\frac{\theta}{\alpha}\right)^{[2]} \frac{(\theta+2 \alpha)^{[n]}}{\theta^{[n]}}-2\left(\frac{\theta}{\alpha}\right)^{2} \frac{(\theta+\alpha)^{[n]}}{\theta^{[n]}}+\left(\frac{\theta}{\alpha}\right)^{(2)}
$$




\section{Asymptotic Distribution}

The $r$ th moment of $K_{n}$ given by (2.6) is written as

$$
E\left[K_{n}^{r}\right]=\sum_{j=0}^{r}(-1)^{r-j}\left(1+\frac{\theta}{\alpha}\right)^{[j]} R\left(r, j, \frac{\theta}{\alpha}\right) \frac{\Gamma(\theta+j \alpha+n) \Gamma(\theta+1)}{\Gamma(\theta+n) \Gamma(\theta+j \alpha+1)} .
$$

By the property of $\Gamma$-function that is $\Gamma(\theta+j \alpha+n) / \Gamma(\theta+n) \approx n^{j \alpha}$ and $R(r, r, \theta / \alpha)=1$ (see (3.12) of Carlitz (1980)), we have the following.

\section{Lemma 3.1}

$$
\mu_{r}^{\prime}=\lim _{n \rightarrow \infty} E\left[\left(\frac{K_{n}}{n^{\alpha}}\right)^{r}\right]=\left(1+\frac{\theta}{\alpha}\right)^{[r]} \frac{\Gamma(\theta+1)}{\Gamma(\theta+1+r \alpha)}=\left(\frac{\theta}{\alpha}\right)^{[r]} \frac{\Gamma(\theta)}{\Gamma(\theta+r \alpha)} .
$$

Specially,

$$
\begin{gathered}
\lim _{n \rightarrow \infty} E\left[\frac{K_{n}}{n^{\alpha}}\right]=\frac{\Gamma(\theta+1)}{\alpha \Gamma(\theta+\alpha)}, \\
\lim _{n \rightarrow \infty} \operatorname{Var}\left[\frac{K_{n}}{n^{\alpha}}\right]=\frac{\Gamma(\theta+1)}{\alpha^{2}}\left\{\frac{\theta+\alpha}{\Gamma(\theta+2 \alpha)}-\frac{\Gamma(\theta+1)}{[\Gamma(\theta+\alpha)]^{2}}\right\}
\end{gathered}
$$

This $\mu_{r}^{\prime}$ is the $r$ th moment of the distribution whose density is given by

$$
\frac{\Gamma(\theta+1)}{\Gamma\left(\frac{\theta}{\alpha}+1\right)} x^{\frac{\theta}{\alpha}} g_{\alpha}(x)
$$

where $g_{\alpha}$ is the density of the Mittag-Leffler distribution with parameter $\alpha$. The density $g_{\alpha}$ is the unique function that satisfies

$$
\int_{0}^{\infty} x^{p} g_{\alpha}(x) d x=\frac{\Gamma(p+1)}{\Gamma(p \alpha+1)}
$$

for any real $p>-1$ (Pitman (1999), p.20-21).

Let $L$ be the random variable which have the density given by (3.1). The relation of the moments with respect to the density given by (3.1) holds for any $\theta / \alpha>-1$. Therefore because of (3.2), the density given by (3.1) is determined uniquely by the moments. Thus, from the point of view of moment, we can get the following.

Proposition 3.2 (Pitman (1999)) $K_{n} / n^{\alpha}$ convenges as $n \rightarrow \infty$ to the distribution with the density given by (3.1), that is, $K_{n} / n^{\alpha} \stackrel{d}{\rightarrow} L$.

We give alternative representations of the distribution whose density is given by (3.1) for some values of parameters. For parameters $\alpha$ and $\theta$ satisfying $0<\alpha \leq 0.5$, $-\alpha<\theta<0$ or $0.5<\alpha<1, \alpha-1 \leq \theta<0$, let $U$ and $W$ be independent random variables such that $W$ has the beta distribution $B e(1+(\theta / \alpha),-\theta / \alpha)$ and $U$ has the generalized Mittag-Leffler distribution with parameters $\alpha, \theta+1$.

The generalized Mittag-Leffler distribution with parameters $0<\alpha<1, \beta(\geq \alpha)$ is a 
distribution whose $r$ th moment is given by $\Gamma(\beta) r ! / \Gamma(\beta+r \alpha)$. The moments determine the distribution uniquely. (Schneider (1996)).

We write $\mu_{r}^{\prime}$ as

$$
\mu_{r}^{\prime}=\frac{\left(1+\frac{\theta}{\alpha}\right)^{[r]}}{r !} \cdot \frac{\Gamma(\theta+1) r !}{\Gamma(\theta+1+r \alpha)} .
$$

If $-1<(\theta / \alpha)<0$, then $(1+(\theta / \alpha))^{[r]} / r$ ! is the $r$ th moment of the beta distribution $B e(1+(\theta / \alpha),-(\theta / \alpha))$. If $0<\alpha \leq 1, \theta+1 \geq \alpha$, then $\Gamma(\theta+1) r ! / \Gamma(\theta+1+r \alpha)$ is the $r$ th moment of the generalized Mittag-Leffler distribution with parameters $\alpha, \theta+1$. Since $0 \leq \alpha<1$ and $\theta>-\alpha$, if $0<\alpha \leq 0.5,-\alpha<\theta<0$ or $0.5<\alpha<1, \alpha-1 \leq \theta<0$, then $\mu_{r}^{\prime}=E\left[(W U)^{r}\right]$.

Thus if $0<\alpha \leq 0.5,-\alpha<\theta<0$ or $0.5<\alpha<1, \alpha-1 \leq \theta<0$, then the distribution with the density given by (3.1) is stochastically equivalent to $W U$.

If $0<\alpha=\theta<1$, then $\mu_{r}^{\prime}=\Gamma(\alpha) r ! \Gamma(\alpha+r \alpha)$, which is the $r$ th moment of the generalized Mittag-Leffler distribution with parameters $\alpha$ and $\alpha$. Therefore, in this case, the distribution with the density given by (3.1) is stochastically equivalent to the generalized Mittag-Leffler distribution with parameters $\alpha$ and $\alpha$.

\section{Marginals of Pitman Sampling Formula}

For Pitman sampling formula given by (1.1), the sum of the right-hand side over all nonnegative integers $m_{1}, m_{2}, \ldots, m_{n}$ satisfying $\sum_{j=1}^{n} j m_{j}=n$ is equal to 1 . Using this property we have for nonnegative integers $r_{1}, \ldots, r_{n}$,

$$
\begin{gathered}
E\left[\prod_{j=1}^{n} M_{j}^{\left(r_{j}\right)}\right]=\frac{\theta^{[r: \alpha]}(\theta+r \alpha)^{[n-s]} n^{(s)}}{\theta \prod^{[n]}} \prod_{j=1}^{n}\left(\frac{(1-\alpha)^{[j-1]}}{j !}\right)^{r_{j}} \\
=\frac{(\theta+\alpha)^{[r-1: \alpha]} \Gamma(\theta+1)}{\Gamma(\theta+r \alpha)} \prod_{j=1}^{n}\left(\frac{(1-\alpha)^{[j-1]}}{j !}\right)^{r_{j}} \frac{\Gamma(\theta+r \alpha+n-s) n^{(s)}}{\Gamma(\theta+n)}
\end{gathered}
$$

where $r=r_{1}+\cdots+r_{n}, s=\sum_{j=1}^{n} j r_{j} \leq n . \quad$ From (4.1), for a fixed $j(=1,2, \ldots)$ and any $r(=0,1,2, \ldots)$ we have

$$
E\left[M_{j}^{(r)}\right]=\frac{\theta^{[r: \alpha]} \Gamma(\theta)}{\Gamma(\theta+r \alpha)}\left(\frac{(1-\alpha)^{[j-1]}}{j !}\right)^{r} \frac{\Gamma(\theta+r \alpha+n-j r) n^{(j r)}}{\Gamma(\theta+n)}
$$

By letting $n$ tend to $\infty$ in the above, we get

$$
\lim _{n \rightarrow \infty} E\left[\frac{j ! M_{j}}{\alpha(1-\alpha)^{[j-1]_{n^{\alpha}}}}\right]^{r}=\left(1+\frac{\theta}{\alpha}\right)^{[r]} \frac{\Gamma(\theta+1)}{\Gamma(\theta+1+r \alpha)}
$$

Using this relation with $r=1,2$, we have for $j=1,2, \ldots$

$$
\lim _{n \rightarrow \infty} E\left[\frac{M_{j}}{n^{\alpha}}\right]=\frac{(1-\alpha)^{[j-1]} \Gamma(\theta+1)}{j ! \Gamma(\theta+\alpha)},
$$




$$
\lim _{n \rightarrow \infty} \operatorname{Var}\left[\frac{M_{j}}{n^{\alpha}}\right]=\Gamma(\theta+1)\left\{\frac{\theta+\alpha}{\Gamma(\theta+2 \alpha)}-\frac{\Gamma(\theta+1)}{[\Gamma(\theta+\alpha)]^{2}}\right\}\left(\frac{(1-\alpha)^{[j-1]}}{j !}\right)^{2} .
$$

Especially,

$$
\begin{gathered}
\lim _{n \rightarrow \infty} E\left[\frac{M_{1}}{n^{\alpha}}\right]=\frac{\Gamma(\theta+1)}{\Gamma(\theta+\alpha)}, \\
\lim _{n \rightarrow \infty} \operatorname{Var}\left[\frac{M_{1}}{n^{\alpha}}\right]=\Gamma(\theta+1)\left\{\frac{\theta+\alpha}{\Gamma(\theta+2 \alpha)}-\frac{\Gamma(\theta+1)}{[\Gamma(\theta+\alpha)]^{2}}\right\} .
\end{gathered}
$$

From (4.1), for a positive integer $l$ and nonnegative integers $r_{1}, \ldots, r_{l}$ we also have

$$
\lim _{n \rightarrow \infty} E\left[\prod_{j=1}^{l}\left(\frac{j ! M_{j}}{\alpha(1-\alpha)^{[j-1]_{n}^{\alpha}}}\right)^{r_{j}}\right]=\mu_{r_{1}+\cdots+r_{1}}^{\prime}
$$

where $\mu_{r}^{\prime}$ is given by Lemma 3.1. Using this relation with $r_{j}=1, r_{t}=1$ for $j, l(=$ $1,2, \ldots, j \neq l)$,

$$
\lim _{n \rightarrow \infty} \operatorname{Cov}\left(\frac{M_{j}}{n^{\alpha}}, \frac{M_{l}}{n^{\alpha}}\right)=\Gamma(\theta+1)\left\{\frac{\theta+\alpha}{\Gamma(\theta+2 \alpha)}-\frac{\Gamma(\theta+1)}{[\Gamma(\theta+\alpha)]^{2}}\right\} \frac{(1-\alpha)^{[j-1]}(1-\alpha)^{[l-1]}}{j ! l !} .
$$

Since $\mu_{r}^{\prime}$ is the $r$ th moment of $L$, by the relation (4.2) we have the following.

Proposition 4.1 For a positive integer l,

$$
\left(\frac{M_{1}}{\alpha n^{\alpha}}, \frac{2 ! M_{2}}{\alpha(1-\alpha)^{[1]_{n} \alpha}}, \cdots, \frac{l ! M_{l}}{\alpha(1-\alpha)^{[l-1]_{n}^{\alpha}}}\right) \stackrel{d}{\rightarrow}(L, L, \ldots, L)
$$

or

$$
\left(\frac{M_{1}}{n^{\alpha}}, \frac{M_{2}}{n^{\alpha}}, \cdots, \frac{M_{l}}{n^{\alpha}}\right) \stackrel{d}{\rightarrow}\left(\alpha L, \frac{\alpha(1-\alpha)^{[1]}}{2 !} L, \ldots, \frac{\alpha(1-\alpha)^{[l-1]}}{l !} L\right) .
$$

Especially, for a positive integer $j$, we have

$$
\frac{M_{j}}{n^{\alpha}} \stackrel{d}{\rightarrow} \frac{\alpha \Gamma(j-\alpha)}{\Gamma(1-\alpha) \Gamma(j+1)} L .
$$

For the random partition $\left(M_{1}, \ldots, M_{n}\right)$ having Pitman sampling formula, let $K_{l ; n}$ be the number of components smaller than or equal to $l(l=1,2, \ldots)$. That is, $K_{l ; n}=M_{1}+\cdots+$ $M_{l}$. Let $S_{l ; n}$ be $1 \times M_{1}+2 \times M_{1} \cdots+l \times M_{l}$. Since $\sum_{j=1}^{l} \alpha(1-\alpha)^{[j-1]} / j !=1-(1-\alpha)^{[l]} / l !$ and $\sum_{j=1}^{l} \alpha(1-\alpha)^{[j-1]} /(j-1) !=\alpha(2-\alpha)^{[l-1]} /(l-1) !$, by (4.3) we have the following.

Proposition 4.2 Let $\left(M_{1}, \ldots, M_{n}\right)$ have Pitman sampling formula given by (1.1) with $0<\alpha<1, \theta\rangle-\alpha$. For a fixed $l(=1,2, \ldots)$,

$$
\frac{K_{l ; n}}{n^{\alpha}} \stackrel{d}{\rightarrow}\left\{1-\frac{(1-\alpha)^{[l]}}{l !}\right\} L
$$

and

$$
\frac{S_{l ; n}}{n^{\alpha}} \stackrel{d}{\rightarrow} \frac{(2-\alpha)^{[l-1]} \alpha}{(l-1) !} L .
$$


It was communicated personally from Prof. Pitman that the statistics of Propositions 4.1 and 4.2 converges almost surely (a.s.) by the reason stated as follows. As the underlying distribution for Pitman sampling formula, we shall consider the twoparameter Poisson-Dirichlet distribution $\left(V_{1}, V_{2}, \ldots\right)$. It has the tails such that $n^{1 / \alpha} V_{n}$ converges to $[L / \Gamma(1-\alpha)]^{1 / \alpha}$ a.s. (Pitman (1997), Pitman and Yor (1997)), where $L$ is the random variable having the density given by (3.1). Therefore by Rouault (1976, 1978), for $j=1,2, \ldots M_{j} / K_{n}$ converges to $\alpha \Gamma(j-\alpha) /[\Gamma(1-\alpha) \Gamma(j+1)]$ a.s. On the other hand, $K_{n} / n^{\alpha}$ converges to $L$ a.s. as stated in Section 1 . Therefore $M_{j} / n^{\alpha}$ converges to $\{\alpha \Gamma(j-\alpha) /[\Gamma(1-\alpha) \Gamma(j+1)]\} L$ a.s. From this convergence we know that the convergences of statistics of Propositions 4.1 and 4.2 hold also almost surely.

\section{Appendix}

For the usual moment $E K_{n}^{r}$, using the relation (2.5) we have for $n=1,2, \ldots$ and $r=1,2, \ldots$

$$
E K_{n}^{r}=\sum_{i=0}^{r}\left\{\begin{array}{c}
r \\
i
\end{array}\right\} E K_{n}^{(i)}=\sum_{j=0}^{r}(-1)^{r-j}\left(1+\frac{\theta}{\alpha}\right)^{[j]} T\left(r, j, \frac{\theta}{\alpha}\right) \frac{(\theta+j \alpha+1)^{[n-1]}}{(\theta+1)^{[n-1]}},
$$

where

$$
T(r, j, \lambda)=\sum_{i=j}^{r}(\lambda+j)^{[i-j]}\left\{\begin{array}{c}
r \\
i
\end{array}\right\}(-1)^{r+i}\left(\begin{array}{l}
i \\
j
\end{array}\right)
$$

For any $y$,

$$
\begin{gathered}
\sum_{j=0}^{r} y^{(j)} T(r, j, \lambda)=\sum_{i=0}^{r}\left\{\sum_{j=0}^{i}\left(\begin{array}{l}
i \\
j
\end{array}\right) y^{(j)}(\lambda+i-1)^{(i-j)}\right\}\left\{\begin{array}{c}
r \\
i
\end{array}\right\}(-1)^{r+i} \\
=\sum_{i=0}^{r}(y+\lambda+i-1)^{(i)}(-1)^{i}\left\{\begin{array}{c}
r \\
i
\end{array}\right\}(-1)^{r}
\end{gathered}
$$

which is equal to $\sum_{i=0}^{r}(-y-\lambda)^{(i)}\left\{\begin{array}{c}r \\ i\end{array}\right\}(-1)^{r}=(-y-\lambda)^{r}(-1)^{r}=(y+\lambda)^{r}$. Thus we have for any $y$,

$$
\sum_{j=0}^{r} y^{(j)} T(r, j, \lambda)=(y+\lambda)^{r}
$$

That is, $T(r, j, \lambda)$ satisfies the relation $(2.7)$ and therefore $T(r, j, \lambda)$ is equal to the function $R(r, j, \lambda)$ of Carlitz (1980). Using this to (5.1) we have the moment given by (2.6).

For the ascending factorial, using the moment given by (2.6) we have for $n=1,2, \ldots$ and $r=1,2, \ldots$

$$
E K_{n}^{[r]}=\sum_{i=0}^{r}\left[\begin{array}{l}
r \\
i
\end{array}\right] E K_{n}^{i}=\sum_{j=0}^{r}(-1)^{r-j}\left(\frac{\theta}{\alpha}\right)^{[j]} I\left(r, j, \frac{\theta}{\alpha}\right) \frac{(\theta+j \alpha)^{[n]}}{\theta^{[n]}}
$$


where

$$
I(r, j, \lambda)=\sum_{i=j}^{r} s(r, i) R(i, j, \lambda)
$$

and $s(r, i)=(-1)^{r-i}\left[\begin{array}{l}r \\ i\end{array}\right]$ is the Stirling number of the first kind.

For any $\mu$,

$$
\sum_{j=0}^{r} I(r, j, \lambda) \mu^{(j)}=\sum_{i=0}^{r}\left(\sum_{j=0}^{i} R(i, j, \lambda) \mu^{(j)}\right) s(r, i)
$$

which is equal to $\sum_{i=0}^{r}(\lambda+\mu)^{i} s(r, i)=(\lambda+\mu)^{(r)}$ by (2.7) and the property of the Stirling number of the first kind $s$. Thus we have for any $\mu$,

$$
\sum_{j=0}^{r} I(r, j, \lambda) \mu^{(j)}=(\lambda+\mu)^{(r)}
$$

Hence if $\lambda \neq 0$ then we have $I(r, j, \lambda)=\left(\begin{array}{l}r \\ j\end{array}\right) \lambda^{(r-j)}$. Using this to (5.2) we have the ascending factorial moment given by (2.8) for $\theta \neq 0$. Also when $\lambda=0, I(r, j, 0)$ equals 1 if $j=r$ and 0 if $j \neq r$.

For $\theta=0$ the relation (5.2) is reduced to

$$
E K_{n}^{[r]}=\sum_{j=0}^{r}(-1)^{r-j} j ! I(r, j, 0) \frac{(j \alpha+1)^{[n-1]}}{(n-1) !}
$$

Hence we get $E K_{n}^{[r]}=(r-1) !(r \alpha)^{[n]} /(n-1) ! \alpha$ which is equal to the evaluation of $E K_{n}^{[r]}$ with $\theta=0$ stated in Section 2.

\section{Acknowledgments}

The authors are deeply grateful to Professor J. Pitman for his valuable comments to the original manuscript. The authors also wish to express their hearty thanks to the referee for his kind comments.

\section{References}

Carlitz, L. (1980), Weighted Stirling numbers of the first and second kind-I, Fibonacci Quart., 18, 147-162.

Engen, S. (1978), Stochastic abundance models with emphasis on biological communities and species diversity, Chapman and Hall Ltd.

Feng, S. and Hoppe, F. (1998), Large deviation principles for some random combinatorial structures in population genetics and Brownian motion, Ann. Appl. Probab., 8, 975-994.

Pitman, J. (1995), Exchangeable and partially exchangeable random partitions, Prob. Theory relat. Fields, 12, 145-158. 
Pitman, J. (1996a), Some developments of the Blackwell-MacQueen urn scheme, in T. S. Ferguson et al., editor,Statistics, Probability and Game Theary; Papers in honor of David Blackwell, Vol. 30 of Lecture Note-Monograph Series, Institute of Mathematical Statistics, Hayward, Ca, 245-267.

Pitman, J. (1996b), Notes on the two parameter generalization of Ewens' random partition structure, Unpublished manuscript.

Pitman, J. (1997), Partition structures derived from Brownian motion and stable subordinators, Bernoulli, 3, 79-96.

Pitman, J. and Yor M. (1997), The two-parameter Poisson-Dirichlet distribution derived from a stable subordinator, Ann. Probab., 25, 855-900.

Pitman, J. (1999), Brownian motion, bridge, excursion and meander characterized by sampling at independent uniform times, Electronic J. Probability, 4, Paper 11, 1-33.

Schneider, W.R. (1996), Completely monotone generalized Mittag-Leffler functions, $E x$ positiones Mathematicae, 14, 3-16.

Rouault, A. (1976), Propriétés asymptotiques d'unn-échantillon d'une variable aléatoire dénombrable connues sous le nom de lois de Zipf", C. R. Acad. Sc. Paris, 283, 379-380.

Rouault, A. (1978), Lois de Zipf et sources markoviennes, Ann. de l'Tnst. Henri Poincaré $B, 14,169-188$.

Yamato, H., Sibuya, M. and Nomachi, T. (1999), Ordered sample from two- parameter GEM distribution, (submitted).

Received March 2,2000 